\title{
Toward A Data Desktop Grid Computing Based On BonjourGrid Meta-Middleware
}

\author{
Walid Saad ${ }^{1}$, Heithem Abbes ${ }^{1,2}$ \\ ${ }^{1}$ LaTICE, Université de Tunis, \\ ESSTT 5, Av. Taha Hussein, B.P. 56, \\ Bab Mnara,Tunis,TUNISIA \\ walid.saad@utic.rnu.tn, mohamed.jemni@fst.rnu.tn
}

\author{
Christophe Cérin ${ }^{2}$,Mohamed Jemni ${ }^{1}$ \\ ${ }^{2}$ LIPN/UMR 7030 - CNRS/Université Paris 13, \\ 99, avenue Jean-Baptiste Clément, \\ 93430 Villetaneuse, FRANCE \\ \{heithem.abbes,christophe.cerin\}@lipn.univ-paris13.fr
}

\begin{abstract}
Desktop Grid or Voluntary Computing systems forms one of the biggest computing platforms in using idles resources over Internet or over LAN's networks. Desktop Grids have been successfully used for solving scientific applications around the world at low cost. However,the data requirements of e-Science applications increase dramatically with the emergence of data-intensive applications. Hence data management becomes one of the major challenge of Desktop Grids. In this work, we describe important challenges that are needed to implementing scalable data-intensive solution in Desktop Grids systems. In addition, we explore the state-of-the art in tools and frameworks for Big Data handling. The proposal solution will be integrated in BonjourGrid Desktop Grid.

Index Terms-Desktop Grids, BonjourGrid, Big Data scheduling, Data Management, Decentralization, Dataintensive application.
\end{abstract}

\section{INTRODUCTION}

Currently, Desktop Grids (DGs) systems evolve in two global areas: Internet Computing (Volunteer Computing) and institution Desktop Grid computing. The first class benefits from exploiting the vast number of idle desktop PCs (volunteers) over Internet. The second, usually called LAN-based DGs, refers to a grid infrastructure that is confined to an institutional boundary, where user participation is governed by the enterprise's policy and desktop PCs are used to support the execution of the enterprise's applications. DGs middlewares such as Condor [12], BOINC [8], PastryGrid [1] [4], XtremWeb [14], OurGrid [9] and GridMP provide researchers a wide range of high throughput computing systems by utilizing idle resources.

In fact,there are many scientific and enterprise applications that deal with a huge amount of data for instance in parallel visualization. In order to process large data-sets, these applications typically need a high performance computing infrastructure. In recent years, highthroughput computing or desktop grid systems perform computational intensive application at low cost. However, since resources in a Desktop Grid are typically accessed through wide area network links, the bottleneck comes with the bandwidth limitation. The issue is to imagine architectures that are able to mask (in part) the bandwidth limitation. According to Fedak and al. in chapter 11 of [13], in Desktop Grid environments, basic data-management tasks such as reliably storing large data-sets are very difficult to accomplish, first because of the volatility of nodes. Second, data privacy and security must be enforced on DGs because we deal with untrusted computers. The data protection mechanism may add non-trivial overhead when processing large volumes of data. Third, since the resources are geographically distributed, the design of a scalable data-intensive solution on these systems is an issue.

In this work, we focus on the latter issue, the other ones are left as part of either the computing or data exchange systems. we propose to build a new Data Desktop Grid system on demand. Our solution focuses on an extension of the BonjourGrid meta-middleware for supporting dataintensive applications by exploiting existing data protocols and middlewares.

Our schema is simple enough to be integrated into major desktop grid platforms. It does not capitalize on the data reuse pattern that can be exploited to achieve better performance. Data re-utilization can be either among tasks of the same particular job or among a succession of job executions of the same or related applications. This issue is left to the data middlewares that we plug in our system. Our work is to coordinate computing and data platforms into a unified system. In this way, we choose a decoupling between the source site and the computation site by the mean of a remote cache and a local cache.

The paper is organized as follows. In section 2 we outline the scientific issues in Data Desktop Grid computing by discussing some related works for data (scheduling, staging, storage and movement). In section 3 we present an overview of the BonjourGrid Meta-middleware. Finally, we describe our data management approach in section 4 and we conclude the paper in section 5 .

\section{RELATED WORK}

In this section we will discuss some of the key technologies being developed in order to prepare an underlying infrastructure for scientist to manage the low-level data handling issues on Grid and Desktop Grid systems. In addition, we present some high-level tools for co-scheduling 
of data and computation in Grid environment.

In recent years, the emergence of data-intensive application has prompted scientists around the world to enable Data Grids. Data-intensive applications include bio-informatics, medical imaging, high energy physics, coastal and environmental modeling and geospatial analysis .Very popular examples of Data Grids are EGEE [33], PPDG [35], Archer [16](Grid appliance [27], [34]), QADPZ [20], distributed.net [28] project and SETI@Home [32]. With over 227,000 active computers in the system (3.1 million total), as of March 2, 2012, SETI@home has the ability to compute over 562 teraFLOPS. For comparison, the K computer [29] was the world's fastest supercomputer, achieved 8162 petaFLOPS. In this way data management is still a challenging issue.

Data storage and movement systems, data scheduling, data-access and meta-data scheme are the main efforts to enable data-intensive applications in Desktop Grid systems. For this reason, Grid Computing researchers have developed several data management tools and distributed file-system technologies. Some projects are based on Client-Server architecture such as GridFTP, SRM [23], Chirp [25], PetaShare [11], Freeloader [26] , while others are run with P2P paradigm like Bitdew [15] , GatorShare [30] and JuxMem [10].

\section{A. Software for data storage and movement in Data Grid}

Several tools are developed to enable Big Data management in conventional Grid Computing. For large data transfer, GridFTP is the most widely used tool through parallel streams. Representative examples of storage systems includes Storage Resource Managers (SRMs), SRB, IBP and NeST have been proven successful in traditional Grid like Globus, TeraGrid and EumedGrid but there are not fitting with the philosophy of DGs. Others systems have targeted Cluster and volatile Desktop to aggregate unused desktop storage.

\section{B. Data storage framework over volatile desktop}

Freeloader [26] framework is designed to aggregate space, and $\mathrm{I} / \mathrm{O}$ bandwidth contributions from volatile desktop storage within a domain to provide a shared cache/scratch space for large, immutable data sets. FreeLoader is designed to handle scientific data under local network.

Farsite [7] builds a secure file system using untrusted desktop computers. It provides file reliability and availability through cryptography, replication and file. Farsite supports the high performance I/O of scientific applications, rather than typical desktop file-I/O workloads in academic environments.

Chirp [25] is a user-level file system for collaboration across distributed systems such as clusters, clouds, and grids. Chirp allows ordinary users to discover, share, and access storage, whether within a single machine room or over a wide area network.

\section{Data Desktop Grid}

Bitdew [15] [19] is an open source data management for Grid, Desktop Grid and Cloud Computing. It can be easily integrated into Grid middlewares (XtremWeb, BOINC, Hadoop, Condor, Glite, Unicore etc..). The BitDew framework offers a programmable environment for enabling data-intensive and long running applications by providing $\mathrm{P} 2 \mathrm{P}$ technologies, DHT for data distribution and BitTorrent for data transfer. In order to manage data, programmers uses a specific APIs to define for every data these 5 abstractions replication, fault-tolerance, lifetime and affinity. BitDew run-time environment manage operations of data creation, deletion, movement, replication, and fault-tolerance operation.

GatorShare [30] is a data management framework that offers a file system interface and an extensible architecture designed to support multiple data transfer protocols, including BitTorrent. for Desktop Grid. It eases the integration with DGs and enables high-throughput data management for unmodified data-intensive applications. For example, in Grid Appliance GatorShare provides an alternative cooperative data distribution service and users are able to download a fully or partial data. GatorShare does not contain mechanisms for replication and fault tolerance compared to Bitdew.

JuxMem (Juxtaposed Memory) [10] is a data sharing service for Grid Computing based on a compromise between DSM systems and P2P systems. It is built over the JXTA middleware and it provides location-transparent data access as well as data persistence in a dynamic, distributed environment. JuxMem features includes data replication, localization and fault tolerance.

\section{Coupling data and computation}

Today, the increasing need to stage large data from user site to the computation site has incited researchers to find mechanisms for co-scheduling of data and computation. Grid users move, manually or employing simple scripts, their data to Grid scratch space from remote archives using transfer tools like FTP and SCP.

Stork [18], a scheduler for data placement activities in a grid environment, is used to schedule data and computation together in Condor and DAGMan. Using Stork input data will be queued, scheduled, monitored, managed, and even check-pointed. Stork provides solutions for data placement problems both in the Grid and Desktop Grid environment since it can interact with different data transfer protocols such as FTP, GridFTP, HTTP and DiskRouter and with . Besides, stork can interact with various data storage systems like SRB and NeST and data management middleware such as SRM.

In [17] PetaShare and Stork are coupled to enable data intensive collaborative science in institutions of Louisiana University by providing additional storage, and infrastructure to access, retrieve and share data. Similarly, Condor and SRM are coupled in [21] to schedule jobs on compute nodes, where data is available. 
In another example, Zhe Zhang and al [31] propose the orchestration of job scheduling with data staging/offloading and on-demand staged data reconstruction in HPC environments.

While the common context is the coordination of jobs and their data, our approach is fundamentally different from these work. We attempt to orchestrate, by extending BonjourGrid Desktop Grid, different data management middlewares and computing systems of Desktop Grid environment to deploy data-intensive applications.

\section{BONJOURGRID META-MidDlEWARE}

In this section we remind the main concepts of the BonjourGrid system and its principal components.

The principal goal of BonjourGrid system [3], [2], [6] is to create, dynamically and in a decentralized way, a specific execution environment for each user to execute any type of applications without any system administrator intervention. Each user, behind a desktop machine in his office, can submit an application. BonjourGrid deploys a master (coordinator), locally on the user machine, and requests for participants (workers). Negotiations to select them should now take place. Using a publish/subscribe infrastructure, each machine publishes its state (idle, worker or master) when changes occur as well as information about its local load or its use cost, in order to provide useful metrics for the selection of participants. Under these assumptions, the master node can select a subset of workers nodes according to the selection criteria. The master and the set of selected workers build the Computing Element (CE) which will execute and manage the user application. When the execution of an application of a CE terminates, its master becomes free, returns in the idle state and it releases all workers before returning to the idle state too. Then, the nodes can participate to others projects.

The key idea of BonjourGrid is to make a meta-grid middleware relying existing Institutional Desktop Grid middleware, and to orchestrate and coordinate multiple instances, i.e multiple CEs, through a publish/subscribe system. Each CE will be owned by the user who has started the master on his machine. Then this CE is responsible for the execution of one or many applications for the same user. As shown on Figure 1, in the user level, a user A (resp. B) deploys his application on his machine and the execution seems to be local. Level 1 (middleware layer) shows that, actually, a CE with 4 (resp. 5) workers has been dynamically created, specifically for the user A (resp. B). Level 0 shows that all machines are interconnected and under the availability of any user.

BonjourGrid is based on three fundamental parts : a) A fully decentralized resources discovery layer, based on Bonjour protocol [24]; b) A CE, using a Desktop Grid middleware such as XtremWeb, Condor or BOINC, which executes and manages the various tasks of applications; $c$ ) A fully decentralized protocol of coordination between a) and b) to manage and control all resources, services and CEs .

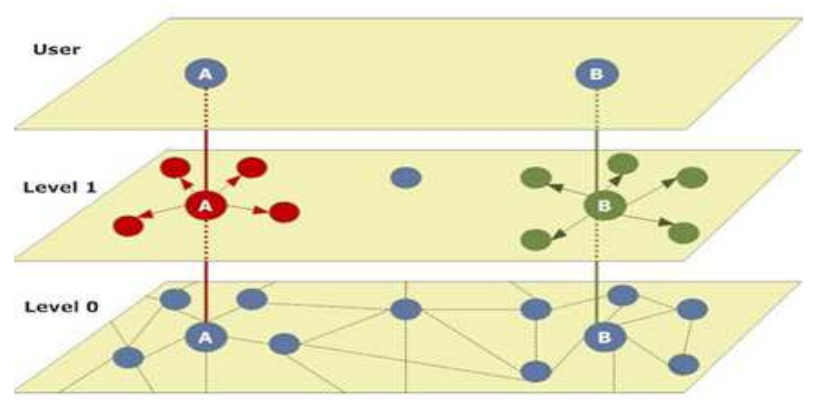

Figure 1. BonjourGrid abstract layers

In addition, we have proposed in [5] a fault tolerant mechanism for BonjourGrid to tolerate the faults of coordinators (see 2). The basic idea is to create, dynamically, $k$ replicas coordinators for each application. We adopt a passive replication technique (where $k$ is a configuration parameter depending on the size of the grid). If a main coordinator fails, a secondary coordinator is resumed. BonjourGrid tries to keep, for each application, $k$ replicas of the coordinator and if there are enough free available machines in the system. Thus, BonjourGrid allocates dynamically a new machine to replace a coordinator 'leaving' the system and if the application is not yet completed.

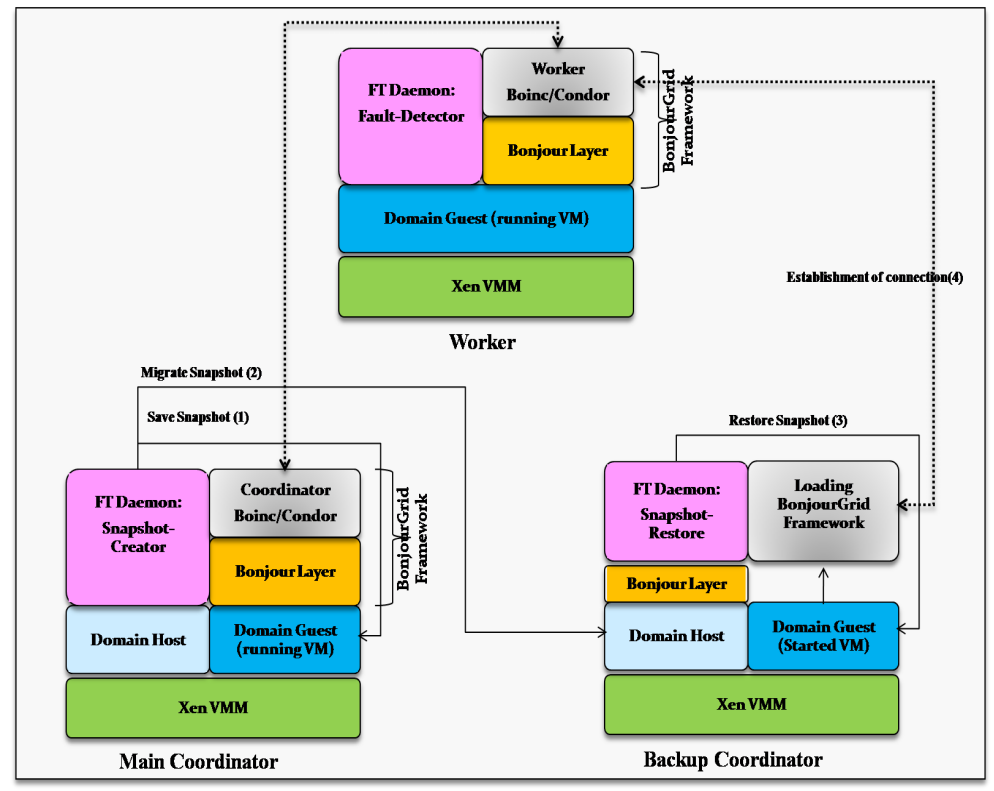

Figure 2. Fault tolerance framework in BonjourGrid.

\section{OVERVIEW OF DESIRED APPROACH}

Why not orchestrate existing data-intensive management system and Grid schedulers for the purpose of Computational Desktop Grid? To answer this question, our solution is focused on extending BonjourGrid metamiddleware to meet the following needs which are not 
met by existing designs. We argue that systems like Stork, Bitdew, GatorShare and Chirp are easily integrated into DGs computing system such as Condor, Boinc and XtremWeb.

\section{A. Purposes}

- Data scheduling. Users get difficulties to implement easily their applications. In fact, to deploy an application, user should place manually, using simple script, the data from its site to the computation platform. To accomplish this task, the user must necessarily have a knowledge about the data transfer protocols such as FTP, SRM tools and Globus GridFTP.

- An efficient high-throughput data management system to support data-intensive applications. In most cases, Desktop Grid middleware lacks dedicated tools for distributing and managing a huge amount of data job. Usually, data and computation are both managed by the job scheduler. This procedure influences negatively on performance, scalability and fault tolerance of the platform. Thus, An ideal Desktop Grid system would allow sophisticated cooperative techniques for data management.

- Enables high-throughput data management for unmodified data-intensive applications. The most important issue is to make unmodified applications available to all participants via BonjourGrid. So, users are not required to modify their binary applications. Also, the proposal platform would deploy bag-oftasks and DAG applications.

- Hiding data management from users and applications. To enable on-demand data management (Staging and scheduling) on Desktop Grid, we plan to build a self-configurable schemes. To ensure this issue, we should provide a high-level API to manage automatically both data queue and job queue within the same framework.

- Data Prefetching. Scientists are already using Grid systems to schedule data intensive applications. These applications are classified into two categories: Bag-Of-Tasks and Computational Work-flow (DAG). Usually, the strategies employed to manage and schedule data in this kind of applications is as follows: 1) Inputs files are staged into computation site before jobs execute 2) Output Files that are produced by jobs are transferred off a worker node to the storage escape of the submitter node. 3) In the case of DAG applications, extra data transfer operations of intermediate output files will be carried out from producer node to consumer node via the submitter node. Motivated by the above facts, we noted that prefetching can be used for optimizing various purposes such as the bandwidth limitation and application performance.

\section{B. Solution design and capabilities}

With the above requirements in mind, the idea consists in creating, dynamically and on demand for each user application, a decentralized data manager in addition to the computing system. The proposed solution supports existing applications without any change in their implementation. The data manager will be initiated and supervised by the BonjourGrid's coordinator node in parallel with the chosen computing middleware (BOINC, XtremWeb or Condor). Thus, BonjourGrid becomes a Meta-Data Grids that orchestrates multiple simultaneous instances of data managers and computational systems. In this section, we present the design of the data manager framework.

We compose the data management framework in two data caches that interact continuously in order to maintain the availability of data throughout the execution of an application. Each cache has a set of services. In figures 3we propose a Remote Cache for data placement activities, in order to reserve the disk space for application and to transfer the required data from the user site to BonjourGrid. Once data are placed on the platform, a second cache called Local Cache is automatically launched to publish and to distribute data over workers [22].

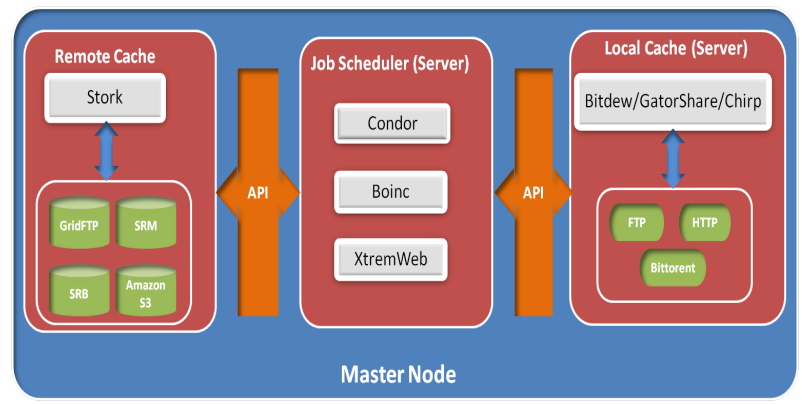

(a) BonjourGrid Coordinator Node

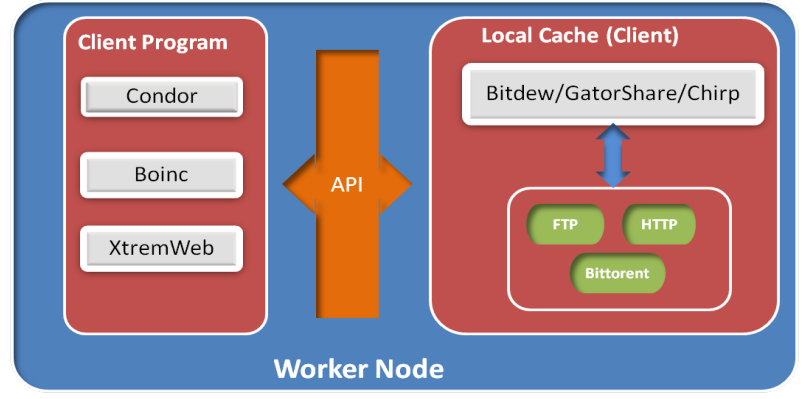

(b) BonjourGrid Worker Node

Figure 3. BonjourGrid new design architecture with Data Manager. The two figures present the interaction between different daemons. Nodes change their status from Idle to Master or Worker. Stork is used as a remote cache for staging data from data sources into BonjourGrid. For locally data distribution, users can select Bitdew, GatorShare or Chirp.

\section{CONCLUSION AND FUture WORKS}

Data management is a key point to perform dataintensive applications of e-Science. In this paper, we have identified the requirement to build a decentralized Data 
Desktop Grid based on BonjourGrid Meta-Middleware. We have discuss existing tools and techniques to manage and schedule Big Data jobs in Grid environment. Further, we have proposed a novel way to coordinate and orchestrate in a transparent and decentralized manner multiple computing systems and data management frameworks into an unified system. Our approach aims to reuse existing protocols and framework such as Bitdew, Chirp and Stork and to hide the difficulties from users to deploy these systems.

Several issues must be taken into account in our future works. The first issue is to evaluate the data management approach using bag-of-tasks and parallel applications with data dependencies. Precisely, with different case studies,we envision to investigate the performance and scalability of BonjourGrid after graft a data management framework. Experimentation will be conducted on Grid'5000 testbed using a large number of machines.

\section{REFERENCES}

[1] Heithem Abbes, Christophe Cérin, and Mohamed Jemni. PastryGrid: decentralisation of the execution of distributed applications in desktop grid. 6th International Workshop on Middleware for Grid Computing - ACM MGC 2008, 1-5 December, Leuven, Belgium.

[2] Heithem Abbes, Christophe Cerin, and Mohamed Jemni. Bonjourgrid as a descentralized scheduler. IEEE APSCC, December 2008.

[3] Heithem Abbes, Christophe Cerin, and Mohamed Jemni. Bonjourgrid: Orchestration of multi-instances of grid middlewares on institutional desktop grids. Parallel and Distributed Processing Symposium, International, 0:1-8, 2009.

[4] Heithem Abbes, Christophe Cérin, and Mohamed Jemni. A decentralized and fault-tolerant desktop grid system for distributed applications. Concurrency and Computation: Practice and Experience, 22(3):261-277, 2010.

[5] Heithem Abbes, Christophe Cérin, Mohamed Jemni, and Walid Saad. Fault tolerance based on the publish-subscribe paradigm for the bonjourgrid middleware. Proceedings of the 2010 11th IEEE/ACM International Conference on Grid Computing, Brussels, Belgium, October 25-29, 2010, pages 57-64, 2010.

[6] Heithem Abbes, Christophe Cérin, Mohamed Jemni, and Walid Saad. Toward a meta-grid middleware. Journal of Internet Technology, Volume 11 No1, 2010.

[7] Atul Adya, William J. Bolosky, Miguel Castro, Gerald Cermak, Ronnie Chaiken, John R. Douceur, Jon Howell, Jacob R. Lorch, Marvin Theimer, and Roger P. Wattenhofer. Farsite: federated, available, and reliable storage for an incompletely trusted environment. SIGOPS Oper. Syst. Rev., 36(SI):1-14, December 2002.

[8] David P. Anderson. Boinc: A system for public-resource computing and storage. Grid Computing, IEEE/ACM International Workshop on, 0:4-10, 2004.

[9] Nazareno Andrade, Walfredo Cirne, Francisco Vilar Brasileiro, and Paulo Roisenberg. Ourgrid: An approach to easily assemble grids with equitable resource sharing. Job Scheduling Strategies for Parallel Processing, 9th International Workshop, JSSPP 2003, Seattle, WA, USA, June 24, 2003, Revised Papers, 2862:61-86, 2003.

[10] Gabriel Antoniu, Luc Bougé, and Mathieu Jan. JuxMem: An Adaptive Supportive Platform for Data Sharing on the Grid. Scalable Computing: Practice and Experience, 6(33):45-55, November 2005.

[11] Mehmet Balman, Ibrahim Suslu, and Tevfik Kosar. Distributed data management with petashare. In Proceedings of ACM SIGAPP 15th Mardi Gras Conference, Baton Rouge, Louisiana, pages 19:1-19:1, 2008.

[12] Ali R. Butt, Rongmei Zhang, and Y. Charlie Hu. A self-organizing flock of condors. J. Parallel Distrib. Comput., 66(1):145-161, 2006.

[13] Christophe Cérin and Gilles Fedak. Desktop grid Computing. ISBN10: 1439862141 ISBN-13: 978-1439862148, 2012.

[14] G. Fedak, C. Germain, V. Neri, and F. Cappello. XtremWeb: a generic global computing system. Cluster Computing and the Grid, 2001. Proceedings. First IEEE/ACM International Symposium on, pages 582-587, 2001.
[15] Gilles Fedak, Haiwu He, and Franck Cappello. BitDew: A data management and distribution service with multi-protocol file transfer and metadata abstraction. Journal of Network and Computer Applications, 32(5):961-975, September 2009.

[16] R. J. Figueiredo, P. O. Boykin, J. A. B. Fortes, T. Li, J.-K. Peir, D. Wolinsky, L. K. John, D. R. Kaeli, D. J. Lilja, S. A. McKee, G. Memik, A. Roy, and G. S. Tyson. Archer: A Community Distributed Computing Infrastructure for Computer Architecture Research and Education. Collaborative Computing: Networking, Applications and Worksharing, 10:70-84, 2009.

[17] Tevfik Kosar and Mehmet Balman. A new paradigm: Data-aware scheduling in grid computing. Future Generation Computer Systems, 25(4):406 - 413, 2009.

[18] Tevfik Kosar and Miron Livny. Stork: Making Data Placement a First Class Citizen in the Grid. Distributed Computing Systems, International Conference on, 0:342-349, 2004.

[19] Mohamed Labidi, Bing Tang, Gilles Fedak, Maher Khemakem, and Mohamed Jemni. Scheduling Data and Task on Data-Driven Master/Worker Platform. The 13th International Conference on Parallel and Distributed Computing, Applications and Technologies (PDCAT-12), IEEE,Beijing, Chine, 2012.

[20] Qadpz - quite advanced distributed parallel zystem. http://qadpz. sourceforge.net/.

[21] Alexandru Romosan, Doron Rotem, Arie Shoshani, and Derek Wright. Co-scheduling of computation and data on computer clusters. Proceedings of the 17th international conference on Scientific and statistical database management, pages 103-112, 2005.

[22] Walid Saad, Heithem Abbes, Christophe Cérin, and Mohamed Jemni. A self-configurable desktop grid system on-demand. Seventh International Conference on P2P, Parallel, Grid, Cloud and Internet Computing, 3PGCIC 2012, Victoria, BC, Canada, November 12-14, 2012, pages 196-203, 2012.

[23] A. Shoshani, A. Sim, and J. Gu. Storage Resource Managers: Middleware Components for Grid Storage. In Nineteenth IEEE Symposium on Mass Storage Systems, 2002.

[24] Daniel Steinberg and Stuart Cheshire. Zero configuration networking: The definitive guide, first edition. o'reilly media,inc. December 2005.

[25] Douglas Thain, Michael Albrecht, Hoang Bui, Peter Bui, Rory Carmichael, Scott Emrich, and Patrick Flynn. Data Intensive Computing with Clustered Chirp Servers. IGI, 2012.

[26] Sudharshan S. Vazhkudai, Xiaosong Ma, Vincent W. Freeh, Jonathan W. Strickland, and et al. Freeloader: Scavenging desktop storage resources for scientific data. In proceeding of supercomputing, 2005.

[27] D. I. Wolinsky and R. J. Figueiredo. Simplifying resource sharing in voluntary grid computing with the grid appliance. Parallel and Distributed Processing, 2008. IPDPS 2008. IEEE International Symposium on, pages 1-8, April 2008.

[28] distributed.net. http://www.distributed.net.

[29] Top500 list. http://www.top500.org.

[30] Jiangyan Xu and Renato J. O. Figueiredo. Gatorshare: a file system framework for high-throughput data management. Proceedings of the 19th ACM International Symposium on High Performance Distributed Computing, HPDC 2010, Chicago, Illinois,USA, June 21-25, 2010, pages 776-786, 2010.

[31] Zhe Zhang, Chao Wang, Sudharshan S. Vazhkudai, Xiaosong Ma, Gregory G. Pike, John W. Cobb, and Frank Mueller. Optimizing center performance through coordinated data staging, scheduling and recovery. SC '07, pages 55:1-55:11, New York, NY, USA, 2007. ACM.

[32] Seti@home.http://setiathome.ssl.berkeley.edu.

[33] Enabling grids for e-science in europe. http://www.eu-egee.org.

[34] Grid appliance. http://www.grid-appliance.org.

[35] Particle physics data grid. http://ppdg.net. 